\title{
Peliosis hepatis mimicking cancer: A case report
}

\author{
WEIDONG DAI and DEWU ZHONG \\ Department of General Surgery, Xiangya Second Hospital, Central South University, Changsha, Hunan 410011, P.R. China
}

Received February 16, 2013; Accepted July 1, 2013

DOI: 10.3892/ol.2013.1479

\begin{abstract}
Peliosis hepatis $(\mathrm{PH})$ is a rare condition characterized by the presence of blood-filled cavities within the liver. The etiology of $\mathrm{PH}$ remains unknown, but it has been reported to be associated with infections or malignancies. However, the cause of $\mathrm{PH}$ is not clear in $20-50 \%$ of patients. The current study presents the case of a 19-year-old male who presented with right upper quadrant pain that had lasted for three days. The patient was a student with no previous medical history. Contrast-enhanced computer tomography (CT) and ultrasonography showed a neoplasm in the right liver and a diagnosis of primary liver cancer was made due to the manifestation of the disease and the results of physical tests. The individual was treated successfully with an irregular right hemihepatectomy and was in good health at 6-months post-surgery. A tissue specimen was obtained and determined to be $\mathrm{PH}$ by pathological examination and immunohistochemistry analysis. Consequently, a diagnosis of PH must be considered in cases like this.
\end{abstract}

\section{Introduction}

Peliosis hepatis (PH) is a rare condition characterized by blood-filled cystic cavities, ranging between $1 \mathrm{~mm}$ and several centimeters in diameter $(1,2)$. The mechanism of $\mathrm{PH}$ is associated with sinusoidal expansion, which is caused by obstructions in the junction of the sinusoidal and central veins of the liver. This results in focal hepatic necrosis, liver sinusoidal barrier destruction and damaged endothelial cells, as red blood cells enter the space of Disse from the sinusoids and form cystic cavities (3). The current study presents the case of a 19-year-old male who complained of right upper quadrant pain that had lasted for three days. The patient was a student with no previous medical history. Contrast enhanced

Correspondence to: Professor Dewu Zhong, Department of Hepatobiliary Surgery, Xiangya Second Hospital, Central South University, Renmin Middle Road, Changsha, Hunan 410011, P.R. China

E-mail: fbron.student@sina.com

Abbreviations: $\mathrm{PH}$, peliosis hepatis; CT, computer tomography

Key words: peliosis hepatis, primary liver cancer, diagnosis computer tomography (CT) and ultrasonography identified a neoplasm in the right liver, which was hypothesized to indicate primary liver cancer by the manifestation of the disease and the physical tests. The patient was treated successfully with an irregular right hemihepatectomy and was in good health at 6-months post-surgery. A tissue specimen was obtained and was determined to be $\mathrm{PH}$ by pathological examination and immunohistochemistry analysis. Written informed consent was obtained from the patient.

\section{Case report}

A 19-year-old male was admitted to Xiangya Second Hospital (Changsha, China) complaining of right upper quadrant pain that had lasted for three days. Enhanced CT and ultrasonography showed a neoplasm of $\sim 3.5 \times 4.5 \times 4.5 \mathrm{~cm}$ in size in the right lobe of the liver (Fig. 1). The periphery of the neoplasm was significantly enhanced in the arterial phase. Laboratory tests were performed with the following results: White blood

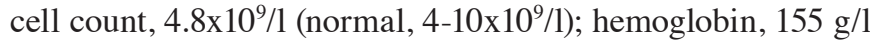
(normal, 110-160 g/1); hematocrit, 47.7\% (normal, 39-52\%); platelet count, $232 \times 10^{9} / 1$ (normal, 100-300 $\times 10^{9} / 1$ ); total bilirubin, $12.6 \mu \mathrm{mol} / 1$ (normal, 5.1-17.1 $\mu \mathrm{mol} / 1$ ); prothrombin time, $12 \mathrm{sec}$ (normal, 11-15 sec); and $\alpha$-fetoprotein, $2.01 \mathrm{mg} / 1$ (normal, <20 mg/l). In addition, electrocardiography and chest X-rays showed no marked abnormalities. The patient had no previous medical history and no history of exposure to toxic agents or drug use. Right upper quadrant pain lasting three days was the only manifestation and the degree of pain was slight, but persistent, and was not accompanied by fever or vomiting. The case was discussed with radiologists due to its specificity and a diagnosis of a hepatocarcinoma was suggested.

Following the pre-operative preparations, surgery was performed under general anesthesia. A mass was identified in the right lobe of the liver, but it did not resemble a hepatocarcinoma and the texture was soft. Mobilization of the right liver lobe was carried out by cutting the ligaments, and prior to cutting the mass, the Pringle maneuver was performed to prevent bleeding. As predicted, the intraoperative frozen pathology of the mass indicated that it was benign. The patient recovered well and was discharged one week later.

The specimen was confirmed to be PH by pathological examination and immunohistochemistry analysis. Microscopically, there were blood filled cystic spaces of variable sizes and hemorrhagic necroses were present adjacent to peliotic spaces without endothelial lining (Fig. 2). Immunohistochemistry tests for CD31, CD34 and SMA were 


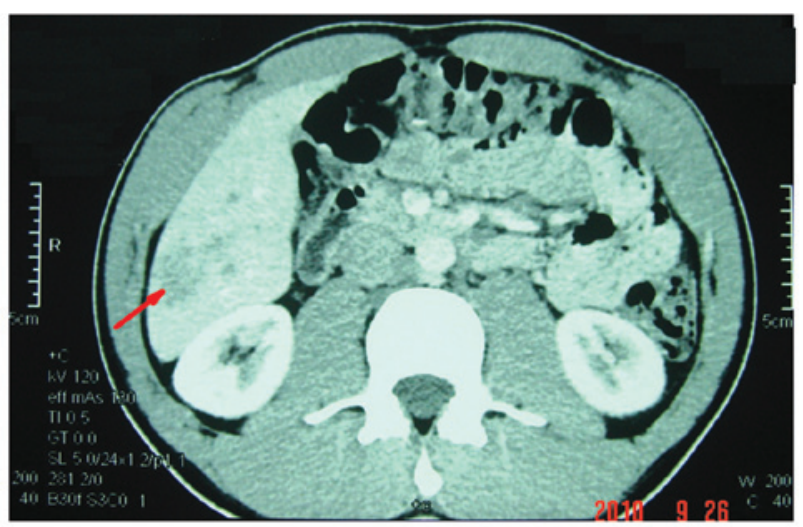

Figure 1. Computed tomography (CT) scan showing the Neoplasm $(\sim 3.5 \times 4.5 \times 4.5 \mathrm{~cm})$ in the right lobe of the liver, as indicated by the arrow

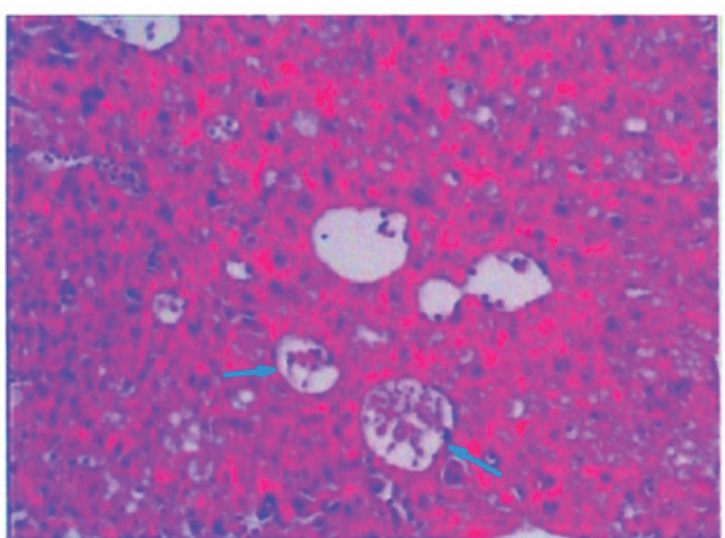

HEx100

Figure 2. Microscopically-determined variably sized blood-filled cystic spaces and hemorrhagic necroses were present adjacent to peliotic spaces without endothelium lining, as indicated by the arrow.

negative in the sinusoidal dilation area, but positive in the normal sinusoidal area.

\section{Discussion}

PH was first described in 1861 by Wagner (4) and named by Schoenlank in 1916 (5). The etiology of $\mathrm{PH}$ remains unknown, but it has been reported to be associated with infectious and non-infectious causes, including drugs, chemicals, bacterial and viral infections and malignancies. Bartonella henselae is hypothesized to be the primary cause of infection (6) and Kitchell et al (7) previously demonstrated that $\mathrm{PH}$ is associated with Bartonella henselae in dogs. In addition, human immunodeficiency virus infection (8) and other wasting diseases $(6,9-11)$ are associated with $\mathrm{PH}$ due to the patients weakened immune system, which directly or indirectly increases the risk of Bartonella henselae infection. However, a previous study reported that $\mathrm{PH}$ in cats is not associated with Bartonella henselae infection (12), indicating that cats have limited value as models for the analysis of Bartonella henselae in PH and that this association must be investigated further. The action of vascular endothelial growth factor has been observed to be important in the pathogenesis of PH (13). Drugs that act against PH include androgenic-anabolic steroids (3), tamoxifen (14), contraceptive steroids (15) and corticosteroids (16). Notably, $\mathrm{PH}$ may present as the cardinal symptom of specific diseases, including Hodgkin's lymphoma (17). However, the causes of $\mathrm{PH}$ have not be identified in $20-50 \%$ of patients (18), as observed in the current case report.

Hepatocellular carcinoma (HCC) is one of the most common types of tumor worldwide, and particularly in China. Enhanced CT is the primary tool used to distinguish $\mathrm{PH}$ from HCC. Commonly, HCC shows hyperattenuation during the arterial phase, with rapid washout during the portal venous phase and iso- or hypoattenuation during the delayed phase. By contrast, during the arterial phase of $\mathrm{PH}$, the lesions usually show early globular enhancement. In addition, multiple small accumulations of contrast material in the center and centrifugal progression of enhancement, without a mass effect on hepatic vessels, is present during the portal venous phase, as determined by enhanced CT. Diffuse increased attenuation may be observed during the delayed phase (18-20). Small lesions (diameter, $>1 \mathrm{~cm}$ ) may not be visible on enhanced CT (21) and magnetic resonance images are atypical for such lesions and the lesions may therefore be confused with hematomas, hemangiomas and HCC (2). PH may not be completely distinguished from HCC and other liver tumors by imaging tests. In the current case report, the patient had no previous medical history and enhanced CT showed a neoplasm mimicking cancer with atypical symptoms and a negative AFP value, making the formation of a diagnosis difficult. In addition, a biopsy was not performed as it could have caused a fatal hemorrhage.

An increasing number of studies have analyzed $\mathrm{PH}$ and possible differential diagnoses include hemangioma, hepatic adenoma, focal nodular hyperplasia, hepatic abscess and hypervascular metastases. In patients with atypical liver lesions, a diagnosis of $\mathrm{PH}$ must be considered, particularly in patients with no previous medical history or identifiable causes. Currently, there are no specific treatments available for $\mathrm{PH}$, however, surgery must be performed on patients with a hemorrhage, long-term medical history or limited lesions. In addition, a liver transplant is necessary when patients have serious accompanying symptoms, including hepatic function failure. In these cases, the termination of any prescribed drugs is vital.

\section{References}

1. Zak FG: Peliosis hepatis. Am J Pathol 26: 1-15, 1950.

2. Iannaccone R, Federle MP, Brancatelli G, et al: Peliosis hepatis: spectrum of imaging findings. AJR Am J Roentgenol 187: W43-W52, 2006.

3. Garcia-Tsao G, Panzini L, Yoselevitz M and West AB: Bacillary peliosis hepatis as a cause of acute anemia in a patient with the acquired immunodeficiency syndrome. Gastroenterology 102: 1065-1070, 1992

4. Wagner E. Ein fall von blutcysten in der leber. Arc Heilkunde 2: 369-370, 1861 (In German).

5. Schoenlank W: Ein fall von peliosis hepatis. Virchows Arch A Pathol Anat 222: 358-364, 1916 (In German).

6. Slater LN, Welch DF and Min KW: Rochalimaea henselae causes bacillary angiomatosis and peliosis hepatis. Arch Intern Med 152: 602-606, 1992.

7. Kitchell BE, Fan TM, Kordick D, Breitschwerdt EB, Wollenberg G and Lichtensteiger CA: Peliosis hepatis in a dog infected with Bartonella henselae. J Am Vet Med Assoc 216: 519-523, 2000 
8. Koehler JE: Bartonella-associated infections in HIV-infected patients. AIDS Clin Care 7: 97-102, 1995.

9. Lie JT: Pulmonary peliosis. Arch Pathol Lab Med 109: 878-879, 1985.

10. Nadell J and Kosek J: Peliosis hepatis. Twelve cases associated with oral androgen therapy. Arch Pathol Lab Med 101: 405-410, 1977.

11. Tsutsumi Y, Ito S, Ichiki K, et al: Systemic amyloidosis complicated with peliosis. Ann Hematol 88: 917-920, 2009.

12. Buchmann AU, Kempf VA, Kershaw O and Gruber AD: Peliosis hepatis in cats is not associated with Bartonella henselae infections. Vet Pathol 47: 163-166, 2010.

13. Edwards R, Colombo T and Greaves P: 'Have you seen this?'peliosis hepatis. Toxicologic Pathol 30: 521-523, 2002.

14. Malet PF and Moonka D: Peliosis hepatis: old disease, new cause. Gastroenterology 101: 864-866, 1991.

15. Zafrani ES, Pinaudeau Y, Le Cudonnec B, Julien M and Dhumeaux D: Focal hemorrhagic necrosis of the liver. A clinicopathological entity possibly related to oral contraceptives. Gastroenterology 79: 1295-1299, 1980.
16. Bagheri SA and Boyer JL: Peliosis hepatis associated with androgenic-anabolic steroid therapy. A severe form of hepatic injury. Ann Intern Med 81: 610-618, 1974.

17. Kleger A, Bommer M, Kunze M, et al: First reported case of disease: peliosis hepatis as cardinal symptom of Hodgkin's lymphoma. Oncologist 14: 1088-1094, 2009.

18. Kim SH, Lee JM, Kim WH, Han JK, Lee JY and Choi BI: Focal peliosis hepatis as a mimicker of hepatic tumors: radiological-pathological correlation. J Comput Assist Tomogr 31: 79-85, 2007.

19. Gouya H, Vignaux O, Legmann P, de Pigneux G and Bonnin A Peliosis hepatis: triphasic helical CT and dynamic MRI findings. Abdom Imaging 26: 507-509, 2001.

20. Savastano S, San Bortolo O, Velo E, Rettore C and Altavilla G: Pseudotumoral appearance of peliosis hepatis. AJR Am J Roentgenol 185: 558-559, 2005.

21. Radin DR and Kanel GC: Peliosis hepatis in a patient with human immunodeficiency virus infection. AJR Am J Roentgenol 156: 91-92, 1991. 